\title{
Effects of resistance and all-round, functional training on quality of life, vitality and depression of older adults living in long-term care facilities: a 'randomized' controlled trial [ISRCTN87 I7728I] Marijke JM Chin A Paw*, Mireille NM van Poppel, Jos WR Twisk and Willem van Mechelen
} \author{
Boechorststraat 71081 BT Amsterdam, The Netherlands \\ Email: Marijke JM Chin A Paw* - m.chinapaw@vumc.nl; Mireille NM van Poppel - mnm.vanpoppel@vumc.nl; \\ Jos WR Twisk - jwr.twisk@vumc.nl; Willem van Mechelen - w.vanmechelen@vumc.nl \\ * Corresponding author
}

Address: Institute for Research in Extramural Medicine \& Department of Public and Occupational Health VU University Medical Center Van der

Published: 02 July 2004

BMC Geriatrics 2004, 4:5 doi:10.1 I86/I47I-23/8-4-5

This article is available from: http://www.biomedcentral.com/|47|-23/8/4/5

(C) 2004 Chin A Paw et al; licensee BioMed Central Ltd. This is an Open Access article: verbatim copying and redistribution of this article are permitted in all media for any purpose, provided this notice is preserved along with the article's original URL.
Received: 03 September 2003

Accepted: 02 July 2004

\begin{abstract}
Background: Regular physical activity may improve different aspects of wellbeing in older people, such as quality of life, vitality and depression. However, there is little experimental evidence to support this assumption. Therefore, we examined the effect of different training protocols on quality of life, vitality and depression of older adults living in long-term care facilities.
\end{abstract}

Methods: Subjects ( $n=173$, aged 64 to 94 years, living in long-term care facilities), were randomized to six months of three different moderate-intensity group exercise training protocols, or to an 'educational' control condition. Exercise consisted of two 45-60-minute training sessions per week of I) resistance training; 2) all-round, functional training; or 3) a combination of both. Perceived health, the Geriatric Depression Scale (GDS), the Vitality Plus Scale (VPS) and the Dementia Quality of Life questionnaire (DQoL) were administered at baseline and after six months.

Results: In the combined training group a small but significant decline was seen in perceived health, DQoL and VPS score compared to the control group.

Conclusions: We conclude that neither strength training nor all-round, functional training of moderate intensity is effective in improving quality of life, vitality or depression of older people living in long-term care facilities.

\section{Background}

Wellbeing consists of many different aspects, such as bodily wellbeing (e.g. pain, energy/fatigue, sleep disturbance), emotional wellbeing (e.g. depression, anxiety, positive affect), self-concept (e.g. self-esteem), and global perceptions of health. It is assumed that habitual exercise may improve subjective wellbeing in older people. However, there is little experimental evidence to support this assumption. Few randomized controlled trials studied the effects of exercise on wellbeing of older people and results are inconsistent. There is no standardized method to measure wellbeing, and all studies focus on different 
aspects of wellbeing. Peel et al. [1] reported a significant improvement in two out of eleven aspects of wellbeing (perceptions of limitations in physical function and emotional problems) after 8 weeks brisk walking and resistance exercise. Two other recent studies $[2,3]$ found no improvement in their measures of well-being after 8 weeks resistance training or 17 weeks all-round, functional training, respectively.

It is also not clear what type and intensity of activity is most effective for wellbeing. Previously, it was believed that psychological change required high-intensity endurance training. King et al. [4] observed a significant decline in anxiety, stress and ratings of perceived change but not in depressive symptoms after 12 months higher intensity group endurance training, higher intensity home endurance training and lower intensity home endurance training. Strength training also seems of benefit for certain measures of well-being. Tsutsumi et al. [5] observed that both high and low intensity strength training improved mood and self-efficacy in older adults. However, Penninx et al. [6] found a significant beneficial effect of endurance training on depressive feelings and behavior of older women, but not from resistance training. Fewer research is available on other types of training such as functional training or ADL training.

Well-being is a subjective measure and refers to the way people feel about their life. The question is whether exercise and/or physical activity can contribute to an enhanced well-being of older adults, and if so, what type of exercise is most effective. Few studies compared different exercise formats with a social control program, and even fewer studies involved the less mobile and less healthy older population. Therefore, the aim of the present study was to examine the effectiveness of three different group-based moderate-intensity exercise protocols on different aspects of wellbeing, i.e. perceived health, quality of life, vitality and depression of older people, living in long-term care facilities.

\section{Methods \\ Study design}

We conducted a 6-month randomized controlled trial of three different moderate-intensity training protocols among older adults living in long-term care facilities, i.e. homes for the aged with services ranging from independent living to skilled nursing. The primary objective of the trial was to study the effects of three different exercise protocols on physical functioning, quality of life, vitality, and depression. In this report we describe the effects on quality of life, vitality and depression. In each of the six homes, subjects were randomly assigned to one of the three exercise conditions or the control condition, an 'educational' program. The random allocation sequence was generated by computer by two independent students, who also assigned participants to their group. The nine couples were randomized together. Random assignment took place after completion of baseline measurements. After the 6-month trial all study participants were allowed to continue either one of the programs as desired. The VU University medical ethics committee has approved the study protocol.

\section{Study population}

Participants of the study were living in six residential and extended care facilities in the North-Western part of The Netherlands (i.e. West-Friesland). In each home all residents were invited to informative meetings organized in the homes. At these informative meetings the design of the study and the interventions were explained in detail. Easy accessibility to the exercise programs and the possibility of voluntary withdrawal from the study were emphasized. The information was also available in brochures for the people to take home. At the end of the meeting, subjects received a form on which they could assert whether they were interested in the study. Those interested in study participation were screened for eligibility based on the following inclusion criteria: 1) aged 65 or older; 2) living in a nursing home or residential care facility; 3 ) able to walk six meters or more (with or without a walking aid); 4) able to comprehend the study procedures; 5) no medical contraindication for study participation; 6) no rapidly progressive or terminal illness; 7) and not moving away from the home within the six months intervention period ( 5 and 6 were evaluated by their general practitioner). Informed consent was obtained from all subjects.

\section{Interventions}

\section{Strength training}

The strength training program was performed twice a week during 6 months in groups of 5 to 7 participants directed by a trained physical therapist and an assistant. In the first 2 weeks, participants were familiarized with the equipment and the technique of the exercises by exercising with minimal resistance. The following weeks, resistance increased until 2 sets of 8-12 repetitions were possible. Resistance was to be increased after the participant could complete 2 sets of 12 repetitions for 2 consecutive sessions. As a warm-up, each exercise was first performed 10-20 repetitions with minimal resistance. Progression was monitored with exercise logs filled out by the supervising physical therapist and assistant. The five exercises were leg press, lattisimus pulldown, biceps curl and triceps press on TechnoGym equipment, and heel raises with dumbbells ( 1 to $5 \mathrm{~kg}$ each), ankle and/or wrist weights ( 1 and $2 \mathrm{~kg}$ per pair). For the heel raises the number of repetitions were increased if the subjects could lift the maximum weight $(2 \times 5 \mathrm{~kg}$ dumbbells $+2 \times 2 \mathrm{~kg}$ 
ankle weights). Sessions lasted 45-60 minutes and closed with stretching exercises. The program was designed to improve muscle strength of major muscle groups of both upper and lower body, important for functional performance on common daily activities.

\section{All-round functional training}

The all-round, functional training program was performed twice a week during 6 months in groups of 7-15 participants, directed by a trained physical therapist and an assistant. The first week was to familiarize participants with the technique of the exercises. All classes started with 5-10 minutes of warm-up activities: walking (whenever possible), exercise-to-music routines, becoming familiar with the equipment. This was followed by 30-35 minutes of skills training in game-like and cooperative activities, such as throwing and catching a ball while standing up and sitting down on a chair, musical chairs and team pursuit races. The cool-down period (5-10 min) consisted of stretching and relaxation activities (e.g. finger and wrist rolls, shoulder rolls, reaching, leg stretches). All exercises could be adjusted to the individual mobility level. The intensity was gradually increased: the number of repetitions increased, exercises were performed more often standing up straight and the use of wrist and ankle weights ( 1 and $2 \mathrm{~kg}$ per pair) was stimulated. The program was designed to improve muscle strength, speed, endurance, coordination and flexibility to improve functional performance of common daily activities. An emphasis was placed on skills training, meaning that the specific activities required for independence in daily activities were practiced. The design and theoretical background of the all-round, functional training program are described in detail elsewhere [7].

\section{Combination}

Subjects in the combination group performed once weekly a complete strength training session and once weekly a complete functional training session.

All three exercise programs were directed by physical therapists who were already working in the homes and thus familiar with working with the study population. The physical therapists were trained by the primary researcher (MCAP). To encourage standardization, the training protocols were extensively described in a manual and the allround, functional training was illustrated on an instructional videotape. The assistants were either volunteers or students.

\section{Control program}

The control program was designed to provide attention, social interaction and was meant to be a 'placebo' intervention. Participants were told that they were assigned to an 'educational' program (i.e. group discussions about topics of interest to older people such as history of the 20 th century, music, relaxation etc.). Sessions were organized 2 days of the week during 6 months for 45-60 minutes in groups of 7-15 participants, supervised by a professional creative therapist.

\section{Measurements}

Data were collected at baseline and after 6 months intervention by three trained research assistants who were blinded to group assignment, according to a standardized protocol.

Information on demographic characteristics, perceived health, quality of life, vitality and depression was obtained in a personal interview at the homes of the study participants. Quality of life was measured by the Dementia Quality of Life Instrument (DQoL)[8]. The DQoL consists of 29 items divided in five sub-scales: aesthetics, belonging, negative affect, positive effect and esteem. The respondent was instructed to choose from one of several 5 -point response scales placed in front of the respondent on a card. A mean score was calculated for the five subscales as well as for the total DQoL ranging from one to five, with a higher score indicating better quality of life. The Vitality Plus Scale (VPS)[9] was specifically designed to measure the accumulated psychological and physical benefits of exercise participation experienced by older adults. The 10-item scale was designed to capture multiple, interrelated aspects of 'feeling good' relevant to the exercise experience e.g. sleep, energy, bodily pain, appetite, stiffness. The respondent was instructed to choose for each item how they were currently feeling on a 5-point response scale placed in front of the respondent on a card. Depression was assessed with the 30 -item version of the Geriatric Depression Scale [10,11].

Furthermore, two single questions 'How would you rate your health' and 'How would you rate your health compared to people of your age' were added. Respondents could choose one answer from a five-point and a threepoint response scale, respectively. For both scales a higher score indicates better health.

Disability was assessed with questions concerning the difficulty with performing 17 Activities of Daily Living (ADL). For each item, the scores were recalculated ranging from 0 (can perform without difficulty or with difficulty but without help) to 1 (can perform only with help or unable to perform).

\section{Statistical analysis}

Data were analyzed using SPSS for Windows (release 7.5.2) and MlwiN (1998, version 1.02.0002). 
Descriptive data are reported for variables of interest (percentage, mean, SD). Analysis of variance and $\mathrm{Chi}^{2}$-tests were used to compare groups at baseline. To evaluate the effects of the interventions multilevel analysis was used. Using this technique, regression coefficients can be adjusted for the clustering of observations within one home that leads to dependency of observations of different subjects within one home. In the multi-level analysis two levels were defined: 1) patient and 2) home. A linear model was used to study the effect of all three interventions on the continuous outcome values. The parameters of interest are the regression coefficients (beta) indicating the effect of the intervention of interest, compared to the control group. In the 'crude' model the outcome value at 6 months was only adjusted for the value at baseline. In the secondary analyses, adjustments were made for gender, age and class attendance. Regression coefficients and 95\% confidence intervals for the basic and the adjusted model are reported.

The trial was designed to randomize 60 subjects to each intervention group, taking into account a dropout percentage of $25 \%$ with an alpha of $5 \%$ and a power of $80 \%$. Primary analyses were conducted by intention-to-treat with participants analyzed according to the initial randomized assignment. Post-hoc secondary analyses were performed including only those subjects who attended at least $75 \%$ of all exercise classes.

\section{Results}

Recruitment of participants and baseline measurements were conducted from August to November 2000. Followup measurements were conducted from March to May 2001. A total of 251 subjects were visited at home and screened to determine eligibility. Of these, 17 did not meet the inclusion criteria, five declined to participate and five died or became ill before the baseline measurements were completed. The 224 remaining subjects were randomly assigned to the four intervention groups (Figure 1). Mean age of these subjects was 81.7, ranging form 64 to 94 . Of the 224 randomized participants, 173 (78\%) completed the study (i.e. agreed with the final data collection visit). General characteristics of this group are shown in Table 1. Their mean age was $81( \pm 5.4)$ years old and the majority $(86 \%)$ lived in a residential care facility. Subjects reported problems with on average three of $17 \mathrm{ADL}$ activities. Dropout of participants was not significantly different among the four groups (strength training $28 \%$, allround, functional training 20\%, combined training $22 \%$ and control group 31\%). The study participants who dropped out were slightly older ( 83 versus 81 years), and were more often male (20 versus $15 \%$ ) and living in nursing homes (23 versus 14\%).
Of the 173 subjects who completed the study, the median attendance to the strength training was $78 \%$ (range: $0-$ 100 ), to the all-round, functional training $70 \%$ (range: $0-$ 100 ) and to the combined training $73 \%$ (range: 7-100). Attendance to the control program was slightly lower (70\%, range: 0-96). No participant was withdrawn for adverse effects, but eight study participants discontinued the intervention because they found that the exercise program was 'too intensive'. From the exercise logs it appeared that compliance to the strength training protocol appeared difficult. Subjects often trained at a lower intensity and increased the intensity at a lower rate than prescribed.

\section{Intention-to-treat analysis}

Perceived health, quality of life, vitality and depression at baseline and after six months intervention are presented in Table 2. At baseline study participants scored their 'perceived health' on average 2.7 on a 5 -point scale $(1=\mathrm{bad}$, $5=$ excellent), and their 'perceived health compared to people of the same age' 2.6 on a 3 point scale $(1=$ worse, $2=$ the same, $3=$ better). These values did not change after 6 months intervention, except in the combined training group where perceived health decreased with 0.3 points (95\% CI:-0.5;-0.1). The mean of the DQoL scores fell above the midpoint of the scale, except for negative affect, which fell below the midpoint. At baseline, the DQoL sumscore and subscores were not significantly different between groups. In all three exercise groups a small but significant decline in DQoL sumscore $(-0.1$ in all three groups) and aesthetics (-0.5, -0.4 and -0.4 points, respectively) was observed. In the combined training group a small but significant decline in positive affect $(-0.2,95 \%$ CI:-3.3;-0.4) and esteem (-0.2, 95\% CI:-3.3;-0.4) was observed as well.

The mean VPS score at baseline was 36.5 out of 50. After the intervention the combined training group had an average decrease of 1.8 points (95\% CI:-3.3;-0.4). The changes in the other groups were small and not significant. The mean GDS score at baseline was 5.8 and did not change after 24 weeks intervention. A GDS score over 10 denotes depressive symptoms.

The differences in quality of life, vitality and depression between the three exercise groups compared to the control group are presented in Table 3 . After adjusting for score at baseline, age, sex and class attendance a significant difference between the strength training and the control group was found in the DQoL aesthetics subscore (adjusted difference: -0.31 , 95\% CI:-0.59;-0.02). The combined training group declined significantly in perceived health (adjusted difference:-0.5, 95\% CI:-0.89;-0.2), DQoL sumscore (adjusted difference:-0.2, 95\% CI:-0.33;-0.08), aesthetics subscore (adjusted difference:-0.3, 95\% CI:-0.58;- 


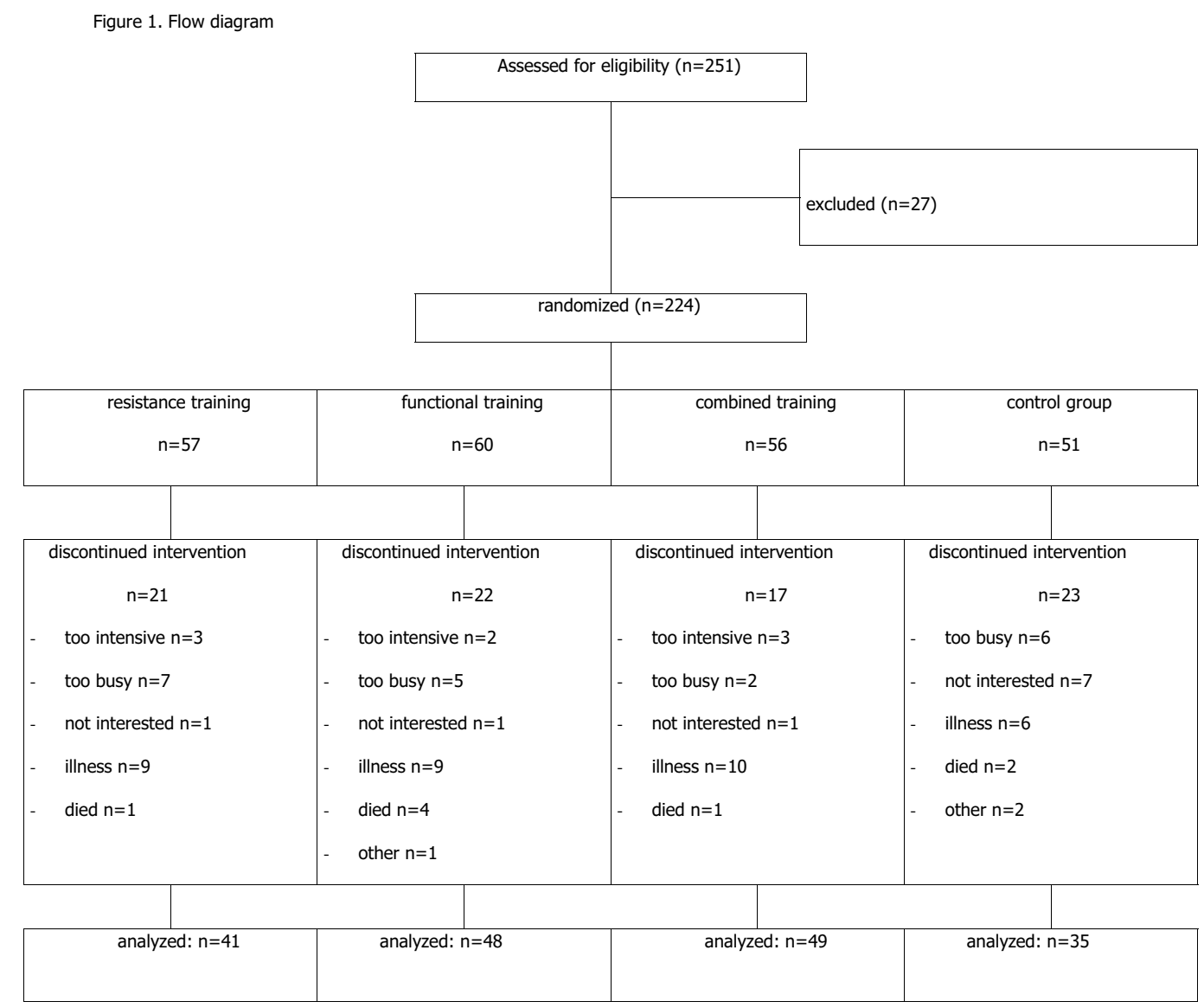

Figure I

Flow diagram.

0.02 ), esteem subscore (adjusted difference:-0.2, 95\% CI: $-0.44 ;-0.04$ ) and VPS score (adjusted difference:-0.5, 95\% CI: $-0.89 ;-0.2)$ compared to the control group. No significant differences between the all-round, functional training group and controls were found.

\section{Subgroup analysis}

We performed post-hoc analyses to examine the effect of the interventions including only those subjects who attended at least $75 \%$ of the exercise classes $(n=74$, i.e. $43 \%$ ) (see Table 4). After adjustment for baseline score, age and sex, no differences between the exercise groups compared to the control group were observed.

\section{Discussion}

Our results suggest that exercise does not affect quality of life, vitality or depression of older adults living in longterm care facilities. After adjustments for baseline score, age, sex and compliance (class attendance), minimal differences in perceived health, quality of life, vitality and depression were observed between the strength training and all-round, functional training group and the nonexercise control group. Noticeable is that the combined training group declined slightly in perceived health, quality of life and vitality compared to the non-exercise controls after 24 weeks. However, in the subgroup that attended at least $75 \%$ of all classes no differences between either of the exercise groups and the controls were seen.

Few randomized controlled trials looking at the psychological effects of exercise in older adults have been performed and all looked at different measures [1-6]. Even fewer studies compared the psychological effects of different types of exercise $[4,6]$. All looked at different training formats (strength training and endurance training), different durations, as well as different aspects of wellbeing 
Table I: General characteristics of elderly living in long-term care facilities $(\mathbf{N}=$ 173)

\begin{tabular}{|c|c|c|c|c|}
\hline & Strength training $(n=4 I)$ & Functional training $(n=48)$ & Combined training $(n=49)$ & Control $(n=35)$ \\
\hline age, mean $\pm S D, y r$ & $81.0( \pm 5.8)$ & $82.1( \pm 4.9)$ & $80.9( \pm 6.3)$ & $81.3( \pm 4.4)$ \\
\hline sex, No. (\%) male/female & $\mathrm{II}(26) / 30(74)$ & $10(21) / 38(79)$ & $8(16) / 41(84)$ & $6(17) / 29(83)$ \\
\hline \multicolumn{5}{|l|}{ marital status, No. (\%) } \\
\hline - married & $9(23)$ & $10(21)$ & $13(27)$ & $\mathrm{II}(3 \mathrm{I})$ \\
\hline - widowed & $27(68)$ & $33(69)$ & $32(65)$ & $23(66)$ \\
\hline - never married & I (3) & $3(6)$ & $3(6)$ & I (3) \\
\hline - divorced & $3(8)$ & $4(2)$ & $\mathrm{I}(2)$ & - \\
\hline \multicolumn{5}{|l|}{$\begin{array}{l}\text { type of residence, No. } \\
(\%)\end{array}$} \\
\hline - nursing home & $6(14)$ & $6(13)$ & $8(16)$ & $4(11)$ \\
\hline - residential care & $35(86)$ & $42(87)$ & $41(84)$ & $31(90)$ \\
\hline $\begin{array}{l}\text { subjects with ADL } \\
\text { disability, No. (\%) }\end{array}$ & $8(22)$ & $8(20)$ & $4(10)$ & $7(21)$ \\
\hline $\begin{array}{l}\text { no. of ADL disabilities, } \\
\text { mean } \pm \text { SD }(0-17)\end{array}$ & $3.3( \pm 2.7)$ & $2.7( \pm 2.3)$ & $3.3( \pm 2.1)$ & $3.1( \pm 2.9)$ \\
\hline $\begin{array}{l}\text { subjects using a walking } \\
\text { aid indoors, No. (\%) }\end{array}$ & $6(15)$ & $8(17)$ & $3(6)$ & $6(17)$ \\
\hline
\end{tabular}

Table 2: Perceived health, quality of life, vitality and depression (mean [95\% $\mathrm{Cl}]$ ) of elderly living in long-term care facilities $(\mathrm{N}=173)$

\begin{tabular}{|c|c|c|c|c|c|c|c|c|}
\hline & \multicolumn{2}{|c|}{ Strength training $(n=4 I)$} & \multicolumn{2}{|c|}{ Functional training $(n=48)$} & \multicolumn{2}{|c|}{ Combined training $(n=49)$} & \multicolumn{2}{|c|}{ Control $(n=35)$} \\
\hline & baseline & $6 \mathrm{~m}$ change & baseline & $6 \mathrm{~m}$ change & baseline & $6 \mathrm{~m}$ change & baseline & $6 \mathrm{~m}$ change \\
\hline $\begin{array}{l}\text { perceived health } \\
(I-5)^{\mathrm{a}}\end{array}$ & $2.3[2.0 ; 2.6]$ & $-0.1[-0.4 ; 0.2]$ & $2.1[1.9 ; 2.3]$ & $0[-0.2 ; 0.3]$ & $2.4[2.1 ; 2.6]$ & $-0.3[-0.5 ;-0.1]^{*}$ & $2.4[2.1 ; 2.7]$ & $0.1[-0.2 ; 0.5]$ \\
\hline $\begin{array}{l}\text { compared health } \\
(I-3)^{\mathrm{a}}\end{array}$ & $2.6[2.4 ; 2.8]$ & $-0.1[-0.4 ; 0.2]$ & $2.4[2.2 ; 2.6]$ & $0[-0.1 ; 0.3]$ & $2.2[2.0 ; 2.5]$ & $0[-0.1 ; 0.3]$ & $2.5[2.3 ; 2.7]$ & $0[-0.2 ; 0.3]$ \\
\hline DqoL $(I-5)^{b}$ & $3.6[3.4 ; 3.7]$ & $-0.1[-0.2 ;-0]^{*}$ & $3.6[3.5 ; 3.7]$ & $-0.1[-0.2 ;-0]^{*}$ & $3.6[3.4 ; 3.7]$ & $-0.1[-0.2 ;-0]^{*}$ & $3.6[3.4 ; 3.7]$ & $0[-0.1 ; 0.1]$ \\
\hline - aesthetics & $4.2[3.9 ; 4.4]$ & $-0.5[-0.7 ;-0.3]^{*}$ & $4.1[3.9 ; 4.3]$ & $-0.4[-0.6 ;-0.2]^{*}$ & $4.0[3.8 ; 4.2]$ & $-0.4[-0.6 ;-0.2]^{*}$ & $4.1[3.9 ; 4.3]$ & $-0.2[-0.5 ; 0]$ \\
\hline - belonging & $3.7[3.5 ; 3.9]$ & $0[-0.2 ; 0.1]$ & $3.7[3.5 ; 3.9]$ & $0[-0.2 ; 0.2]$ & $3.7[3.5 ; 3.9]$ & $0[-0.2 ; 0.2]$ & $3.6[3.3 ; 3.9]$ & $0.2[-0 ; 0.4]$ \\
\hline - negative affect & $2.7[2.5 ; 2.9]$ & $0[-0 ; 0.2]$ & $2.7[2.5 ; 2.8]$ & $0[-0.2 ; 0]$ & $2.6[2.5 ; 2.8]$ & $0[-0.2 ; 0]$ & $2.6[2.4 ; 2.8]$ & $0[-0 ; 0.2]$ \\
\hline - positive affect & $3.7[3.5 ; 3.9]$ & $0[-0.2 ; 0]$ & $3.8[3.6 ; 4.0]$ & $-0.1[-0.3 ; 0]$ & $3.8[3.6 ; 3.9]$ & $-0.2[-0.3 ;-0]^{*}$ & $3.7[3.5 ; 3.9]$ & $0[-0.2 ; 0.1]$ \\
\hline - esteem & $3.7[3.5 ; 3.9]$ & $0[-0.3 ; 0.1]$ & $3.8[3.6 ; 4.0]$ & $0[-0.2 ; 0.1]$ & $3.7[3.6 ; 3.9]$ & $0.2[-0.3 ;-0]^{*}$ & $3.8[3.6 ; 4.0]$ & $0[-0.2 ; 0.1]$ \\
\hline $\begin{array}{l}\text { Vitality Plus } \\
\text { Scale }(10-50)^{c}\end{array}$ & $36.4[34.3 ; 38.5]$ & $0.6[-0.9 ; 2.0]$ & $36.2[34.5 ; 37.9]$ & $0.3[-1.2 ; 1.7]$ & $35.8[33.8 ; 37.8]$ & $-1.8[-3.3 ;-0.4]^{*}$ & $38.0[35.6 ; 40.3]$ & $-0.8[-2.2 ; 0.7]$ \\
\hline GDS $(0-30)^{d}$ & $6.0[4.5 ; 7.6]$ & $0.3[-0.5 ; 1.1]$ & $5.6[4.3 ; 6.8]$ & $-0.3[-1.0 ; 0.5]$ & $6.1[4.8 ; 7.5]$ & $0.3[-0.8 ; 1.4]$ & $5.2[4.0 ; 6.4]$ & $0.2[-0.7 ; 1.1]$ \\
\hline
\end{tabular}

a higher scores indicate better health ${ }^{b}$ higher scores indicate better QoL c higher scores indicate more vitality ${ }^{\mathrm{d}}$ higher scores indicate more depressive symptoms $* \mathrm{p}<0.05$, for within group change from baseline value

such as depression, mood and anxiety, stress and self-efficacy. Findings on outcome measures were inconsistent.

Most studies used a non-exercise or waiting list control group. To positively rule out social interaction or staff attention as the most important underlying factor of improvement for quality of life, vitality and depression we added a sedentary, social activity control group. This may be one explanation for the lack of effect of the exercise programs in our study. The control program may have lead to improvement. King et al. [4] compared higher intensity group, higher intensity home and lower intensity home endurance exercise. Their results suggest that change in physical fitness and social support from group training was not necessary to achieve psychological change.

Another explanation for the lack of effect may be that some aspects of quality of life, vitality and depression represent stable personality traits rather than modifiable states or that the used measures were insufficiently responsive to change. The quantification of psychological wellbeing is difficult. There are many instruments that measure different aspects of wellbeing, such as bodily wellbeing (e.g. pain, energy/fatigue, sleep disturbance), emotional wellbeing (e.g. depression, anxiety, positive 
Table 3: Results of Multilevel analyses regarding the effect of strength training, functional training and the combined training on perceived health, quality of life, vitality and depression of elderly living in long-term care facilities $(\mathbf{N}=\mathbf{1 7 3})$

\begin{tabular}{|c|c|c|c|}
\hline & $\begin{array}{l}\text { Strength training versus } \\
\text { control difference }[95 \% \mathrm{Cl}]\end{array}$ & $\begin{array}{l}\text { Functional training versus } \\
\text { control difference }[95 \% \mathrm{Cl}]\end{array}$ & $\begin{array}{l}\text { Combined training versus } \\
\text { control difference }[95 \% \mathrm{Cl}]\end{array}$ \\
\hline \multicolumn{4}{|l|}{ perceived health } \\
\hline adjusted for score at baseline & $-0.32[-0.69 ; 0.04]$ & $-0.21[-0.57 ; 0.14 ;]$ & $-0.50[-0.85 ;-0.14]^{*}$ \\
\hline $\begin{array}{l}\text { adjusted for score at baseline, } \\
\text { age, sex and compliance }\end{array}$ & $-0.35[-0.72 ; 0.02]$ & $-0.24[-0.60 ; 0.12]$ & $-0.52[-0.88 ;-0.16]^{*}$ \\
\hline \multicolumn{4}{|l|}{ DQoL } \\
\hline adjusted for score at baseline & $-0.13[-0.26 ;-0.004]^{*}$ & $-0.12[-0.24 ; 0.01]$ & $-0.18[-0.30 ;-0.05]^{*}$ \\
\hline $\begin{array}{l}\text { adjusted for score at baseline, } \\
\text { age, sex and compliance }\end{array}$ & $-0.14[-0.27 ; 0.01]$ & $-0.12[-0.25 ; 0.003]$ & $-0.20[-0.33 ;-0.08]^{*}$ \\
\hline \multicolumn{4}{|l|}{ - aesthetics } \\
\hline adjusted for score at baseline & $-0.30[-0.59 ;-0.01]^{*}$ & $-0.25[-0.53 ; 0.03]$ & $-0.29[-0.57 ;-0.01]^{*}$ \\
\hline $\begin{array}{l}\text { adjusted for score at baseline, } \\
\text { age, sex and compliance }\end{array}$ & $-0.31[-0.59 ;-0.02]^{*}$ & $-0.24[-0.52 ; 0.04]$ & $-0.30[-0.58 ;-0.02]^{*}$ \\
\hline \multicolumn{4}{|l|}{ - belonging } \\
\hline adjusted for score at baseline & $-0.22[-0.46 ; 0.01]$ & $-0.16[-0.38 ; 0.07]$ & $-0.18[-0.40 ; 0.05]$ \\
\hline $\begin{array}{l}\text { adjusted for score at baseline, } \\
\text { age, sex and compliance }\end{array}$ & $-0.23[-0.46 ; 0.004]$ & $-0.17[-0.40 ; 0.05]$ & $-0.21[-0.43 ; 0.02]$ \\
\hline \multicolumn{4}{|l|}{ - negative affect } \\
\hline adjusted for score at baseline & $-0.02[-0.19 ; 0.16]$ & $-0.10[-0.27 ; 0.06]$ & $-0.13[-0.29 ; 0.04]$ \\
\hline $\begin{array}{l}\text { adjusted for score at baseline, } \\
\text { age, sex and compliance }\end{array}$ & $-0.02[-0.19 ; 0.15]$ & $-0.10[-0.26 ; 0.06]$ & $-0.14[-0.30 ; 0.03]$ \\
\hline \multicolumn{4}{|l|}{ - positive affect } \\
\hline adjusted for score at baseline & $-0.01[-0.21 ; 0.18]$ & $-0.05[-0.23 ; 0.14]$ & $-0.12[-0.31 ; 0.07]$ \\
\hline $\begin{array}{l}\text { adjusted for score at baseline, } \\
\text { age, sex and compliance }\end{array}$ & $-0.05[-0.24 ; 0.14]$ & $-0.07[-0.25 ; 0.12]$ & $-0.17[-0.35 ; 0.02]$ \\
\hline \multicolumn{4}{|l|}{ - esteem } \\
\hline adjusted for score at baseline & $-0.11[-0.32 ; 0.10]$ & $-0.02[-0.22 ; 0.18]$ & $-0.19[-0.39 ; 0.02]$ \\
\hline $\begin{array}{l}\text { adjusted for score at baseline, } \\
\text { age, sex and compliance }\end{array}$ & $-0.13[-0.33 ; 0.08]$ & $-0.05[-0.24 ; 0.15]$ & $-0.24[-0.44 ;-0.04]^{*}$ \\
\hline \multicolumn{4}{|l|}{ - Vitality Plus Scale } \\
\hline adjusted for score at baseline & $0.83[-1.23 ; 2.89]$ & $0.53[-1.47 ; 2.52]$ & $-1.66[-3.65 ; 0.33]$ \\
\hline $\begin{array}{l}\text { adjusted for score at baseline, } \\
\text { age, sex and compliance }\end{array}$ & $0.51[-1.55 ; 2.57]$ & $0.38[-1.60 ; 2.35]$ & $-2.06[-4.05 ;-0.07]^{*}$ \\
\hline \multicolumn{4}{|l|}{ - GDS } \\
\hline adjusted for score at baseline & $-0.004[-\mid .33 ; 1.32]$ & $-0.40[-1.67 ; 0.88]$ & $0.24[-1.03 ; 1.52]$ \\
\hline $\begin{array}{l}\text { adjusted for score at baseline, } \\
\text { age, sex and compliance }\end{array}$ & $0.09[-1.25 ; 1.42]$ & $-0.37[-1.65 ; 0.90]$ & $0.34[-0.95 ; 1.63]$ \\
\hline
\end{tabular}

$*_{\mathrm{p}}<0.05$, for exercise versus control group

Table 4: Results of Multilevel analyses regarding the effect of strength training, functional training and the combined training on perceived health, quality of life, vitality and depression of the subgroup who attended at least $75 \%$ of all exercise classes $(\mathrm{N}=74)$

\begin{tabular}{lccc}
\hline & $\begin{array}{c}\text { Strength training versus } \\
\text { control difference [95\% Cl] }\end{array}$ & $\begin{array}{c}\text { Functional training versus } \\
\text { control difference [95\% CI] }\end{array}$ & $\begin{array}{c}\text { Combined training versus } \\
\text { control difference [95\% Cl] }\end{array}$ \\
\hline perceived health & $-0.003[-0.6 ; 0.6]$ & $0.3[-0.3 ; 0.9]$ & $-0.1[-0.7 ; 0.5]$ \\
DQoL sumscore & $-0.2[-0.4 ; 0.01]$ & $-0.1[-0.3 ; 0.1]$ & $-0.2[-0.4 ; 0.03]$ \\
- aesthetics & $-0.4[-0.8 ; 0.01]$ & $-0.2[-0.7 ; 0.2]$ & $-0.2[-0.7 ; 0.2]$ \\
- belonging & $-0.4[-0.7 ; 0.01]$ & $-0.2[-0.6 ; 0.1]$ & $-0.3[-0.7 ; 0.02]$ \\
- negative affect & $0.1[-0.2 ; 0.5]$ & $0.1[-0.2 ; 0.5]$ & $0.04[-0.3 ; 0.4]$ \\
- positive affect & $-0.2[-0.5 ; 0.2]$ & $-0.04[-0.4 ; 0.3]$ & $-0.1[-0.4 ; 0.2]$ \\
- esteem & $-0.2[-0.6 ; 0.2]$ & $-0.1[-0.4 ; 0.3]$ & $-0.3[-0.7 ; 0.1]$ \\
Vitality Plus Scale & $0.4[-3.0 ; 3.9]$ & $0.4[-3.0 ; 3.8]$ & $-1.4[-4.8 ; 2.1]$ \\
GDS & $-1.1[-3.3 ; 1.0]$ & $-1.5[-3.6 ; 0.7]$ & $-1.3[-3.4,0.9]$ \\
\hline
\end{tabular}

Note: All values are adjusted for score at baseline, age and sex. 
affect), self-concept (e.g. self-esteem), and global perceptions of health. Most instruments are not developed for measuring change over time. Thus, it is important to develop useful instruments in these potentially important areas. The Vitality Plus Scale used in our study was specifically developed to measure perceived psychophysical benefits of exercise participation, but did not indicate any changes in our trial. The responsiveness to change of this scale has been studied insufficiently up till now.

Our study was conducted in the less mobile and less healthy group of older people living in long-term care facilities. This group is likely to experience lower psychological wellbeing because of problems with health, mobility, and autonomy. Therefore, we expected greater gains in wellbeing from exercise. Still, average scores at baseline were not exceptionally low, leaving little room for improvement (ceiling effect). Because of these health and mobility problems, regular exercising at a considerable intensity appeared difficult. Most persons were not used to exercising and afraid to exercise at moderate intensity, which was in some people associated with pain. From the exercise logs it appeared that compliance to the strength training protocol appeared difficult.

The exercise programs showed little improvement in fitness and function (data not shown). In the subgroup of subjects who attended at least $75 \%$ of the classes, improvements in some fitness and function measures were observed (reaction time, eye-hand-coordination, chair rise performance) in the functional training group and the combined training group. No effect of strength training was observed. None of the exercise protocols were effecting ADL-disability (manuscript submitted for publication). Despite the lack of functional improvement subjects may still experience psychological benefit from participating in an exercise program, for instance because of hormonal or metabolic adaptations, alterations in brain monoamines or opioid peptides, the opportunity of socializing, enhanced feelings of competency, or distraction of day-to-day stressors. The studies of King et al. [4] and Tsutsumi et al. [5] suggest that neither high intensities, nor substantial fitness changes are essential to achieve positive mental health effects. Though, both studied healthy community-dwelling older people who were much younger (mean age 57 and 68, respectively).

A limitation of this type of study examining the effect of exercise programs in more real-life circumstances is the difficulty in standardizing and measuring the intensity of the training. Therefore, we cannot say whether the lack of effect is due to the insufficient compliance of the participants or due to the insufficient intensity or frequency of the exercise program.
Another limitation is that we used multilevel analysis. This technique is the state of the art for analyzing discrete variables in this type of trials. However, in our study we used ordinal outcome variables. Therefore, the results have to be interpreted with caution.

We conclude that neither strength training nor all-round, functional training of moderate intensity is effective in improving psychological quality of life, vitality and depression of older people living in long-term care facilities.

\section{Competing interests}

None declared.

\section{Authors' contributions}

MCAP conceived of the study, participated in its design and coordination and performed the statistical analysis. MvP participated in the coordination of the study. JT participated in and advised on the statistical analysis of the study. WvM conceived of the study and participated in the design of the study. All authors read and approved the final manuscript.

\section{Acknowledgements}

The study was financially supported by the Dutch Health Research Council (grant number 2200.0060), the 'Stichting Ouderen in Beweging West-Friesland', Regional Health Care Insurance Company Univé, TechnoGym Benelux B.V. and Nijha Lochem B.V.

\section{References}

I. Peel C, Utsey C, MacGregor J: Exercise Training for Older Adults With Limitations in Physical Function. J Aging Phys Act 1999, 7:62-75.

2. Perrig-Chiello P, Perrig WJ, Ehrsam R, Staehelin HB, Krings F: The effects of resistance training on well-being and memory in elderly volunteers. Age Ageing 1998, 27(4):469-475.

3. Chin A Paw MJM, de Jong N, Schouten EG, van Staveren WA, Kok FJ: Physical exercise or micronutrient supplementation for the wellbeing of the frail elderly? A randomised controlled trial. Br J Sports Med 2002, 36(2): I 26-I3I.

4. King AC, Taylor CB, Haskell WL: Effects of differing intensities and formats of 12 months of exercise training on psychological outcomes in older adults. Health Psychol 1993, I 2(4):292-300.

5. Tsutsumi T, Don BM, Zaichkowsky LD, Delizonna LL: Physical fitness and psychological benefits of strength training in community dwelling older adults. Appl Human Sci 1997, I 6(6):257-266.

6. Penninx BW, Rejeski WJ, Pandya J, Miller ME, Di Bari M, Applegate WB, Pahor M: Exercise and depressive symptoms: a comparison of aerobic and resistance exercise effects on emotional and physical function in older persons with high and low depressive symptomatology. J Gerontol B Psychol Sci Soc Sci 2002, 57(2): I24- I32.

7. Chin A Paw MJM, de Jong N, Stevens M, Bult P, Schouten EG: Development of an exercise program for frail elderly. J Aging and Physical Activity 2001, 9:476-489.

8. Brod M, Stewart AL, Sands L, Walton P: Conceptualization and measurement of quality of life in dementia: the dementia quality of life instrument (DQoL). Gerontologist 1999, 39(I):25-35.

9. Myers AM, Malott OW, Gray E, Tudor-Locke C, Ecclestone NA, Cousins SO, Petrella RI: Measuring accumulated health-related 
benefits of exercise participation for older adults: the Vitality Plus Scale. J Gerontol 1999, 54(9):456-66.

10. Yesavage JA, Brink TL, Rose TL, Lum O, Huang V, Adey M, Leirer VO: Development and validation of a geriatric depression screening scale: a preliminary report. J Psychiatr Res 1982, I7(1):37-49.

II. McGivney SA, Mulvihill M, Taylor B: Validating the GDS depression screen in the nursing home. J Am Geriatr Soc 1994, 42(5):490-2.

\section{Pre-publication history}

The pre-publication history for this paper can be accessed here:

http://www.biomedcentral.com/1471-2318/4/5/prepub

Publish with Biomed Central and every scientist can read your work free of charge

"BioMed Central will be the most significant development for disseminating the results of biomedical research in our lifetime. "

Sir Paul Nurse, Cancer Research UK

Your research papers will be:

- available free of charge to the entire biomedical community

- peer reviewed and published immediately upon acceptance

- cited in PubMed and archived on PubMed Central

- yours - you keep the copyright

Submit your manuscript here:

http://www.biomedcentral.com/info/publishing_adv.asp 